\title{
Netrin-1 interference potentiates epithelial-to-mesenchymal transition through the PI3K/AKT pathway under the hypoxic microenvironment conditions of non-small cell lung cancer
}

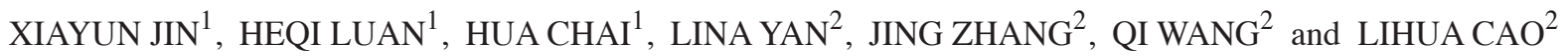 \\ Departments of ${ }^{1}$ Clinical Medicine and ${ }^{2}$ Respiratory Medicine, \\ The Second Hospital Affiliated to Dalian Medical University, Dalian, Liaoning 116027, P.R. China
}

Received March 23, 2018; Accepted January 8, 2019

DOI: $10.3892 /$ ijo.2019.4716

\begin{abstract}
Netrin-1 is overexpressed in several types of cancer. However, whether netrin-1 can potentiate hypoxia-induced tumor progression in lung cancer has not been reported to date. Thus, the objective of the present study was to investigate whether netrin-1 regulates cancer cell migration and invasion under hypoxic conditions in lung cancer and explore the underlying mechanism. A three-dimensional microfluidic chip was used to observe real-time changes in cancer cells, and cobalt chloride $\left(\mathrm{CoCl}_{2}\right)$ was used to simulate a hypoxic microenvironment. Netrin-1 siRNA was employed in the A549 and PC9 cell lines to downregulate the expression of netrin-1. Microfluidic chip, wound healing and Transwell assays were used to examine cell migration and invasion. The expression levels of E-cadherin and vimentin were detected by western blotting. The data demonstrated that netrin-1 mediated epithelial-to-mesenchymal transition (EMT) of A549 and PC9 cells in vitro, which may be associated with the phosphoinositide 3 kinase/AKT pathway. This effect of netrin-1 on the EMT was not observed in the normoxic microenvironment. In this retrospective study, netrin-1 concentrations were evaluated in serum obtained from patients with non-small cell lung cancer (NSCLC) and compared with healthy control samples by quantitative enzyme-linked immunosorbent analysis. The serum concentration of netrin-1 was found to be significantly higher in NSCLC patients compared with that in healthy donors. Taken together, the findings of the present study highlight a novel role for netrin-1
\end{abstract}

Correspondence to: Professor Lihua Cao or Professor Qi Wang, Department of Respiratory Medicine, The Second Hospital Affiliated to Dalian Medical University, 467 Zhong shan Road, Dalian, Liaoning 116027, P.R. China

E-mail: 13898680918@163.com

E-mail:wqdlmu@163.com

Key words: netrin-1, epithelial-to-mesenchymal transition, non-smal cell lung cancer in tumor development under hypoxia in NSCLC and provide further evidence for the use of netrin-1 as a therapeutic target.

\section{Introduction}

Lung cancer has the highest morbidity and mortality rates among malignant tumors globally (1). Although a number of advanced technologies and high-precision testing methods have been applied in the detection and treatment of lung cancer in recent decades, the 5-year survival rate among patients with invasive and metastatic lung cancer remains low (2-6). Hypoxia, a hallmark of cancer development that occurs mainly in fast-growing solid tumors, is characterized as closely associated with cell metastasis (7-9) and may be a major contributor to tumor progression and poor clinical outcomes (10-12). Hypoxia is known to trigger epithelial-to-mesenchymal transition (EMT) in lung cancer cells, but a clearer understanding of the underlying mechanism is required for the development of treatments targeting this process.

Netrin-1, the best-characterized ligand of the netrin family, is a highly conserved cell-secreted soluble protein. Accumulating evidence has confirmed that netrin-1 is not only involved in the development of the central nervous system (13-17), but also plays a completely different role regulating the adhesion and proliferation of multiple cancer cell types (18-20). In recent studies, the overexpression of netrin-1 has been considered as a selective advantage for tumor progression in several human cancers, such as colorectal cancer, metastatic breast cancer and neuroblastoma $(18,21,22)$. Moreover, Gibert and Mehlen proposed that monoclonal antibodies to netrin-1 can block ligand-receptor interaction with dependence receptors, thereby inducing tumor cell apoptosis (23). It has been demonstrated that FAK is an important downstream molecule of netrin-1 that interacts within the phosphoinositide 3 kinase (PI3K)/AKT and mitogen-activated protein kinase (MAPK) pathways to induce EMT (24-26). Moreover, netrin-1 was linked to the p38 MAPK and PI3K/AKT signaling pathways in experiments of netrin-1-mediated induction of Schwann cell migration in peripheral nervous system injury (19). However, additional research is required to determine whether netrin-1 activation is directly associated with hypoxia and EMT. 
The aim of present study was to investigate whether netrin-1 is involved in the hypoxia-induced EMT of non-small cell lung cancer (NSCLC) cells. The concentration of netrin-1 in the serum of NSCLC patients was compared with that in the healthy control group in order to determine whether netrin-1 is associated with lung cancer progression.

\section{Materials and methods}

Cell culture and simulation of hypoxic conditions. The human NSCLC cell lines A549, NCI-H1299, NCI-H1975, SPC-A1, PC9 and NCI-H522, along with the normal human bronchial epithelial cell line 16-HBE, were obtained from the Cell Bank of Type Culture Collection of Chinese Academy of Sciences (Shanghai, China). The cells were cultured in RPMI-1640 medium (Gibco, Invitrogen; Thermo Fisher Scientific, Inc., Carlsbad, CA, USA) containing 10\% fetal bovine serum (FBS; Thermo Fisher Scientific, Inc.) at $37^{\circ} \mathrm{C}$ in a humidified atmosphere containing $5 \% \mathrm{CO}_{2}$. To simulate a hypoxic microenvironment in vitro, the cells were treated with $100 \mu \mathrm{mol} / 1 \mathrm{CoCl}_{2}$ (Sigma-Aldrich; Merck KGaA, St. Louis, MO, USA) for $48 \mathrm{~h}$.

Cell transfection with small interfering (si)RNA. A549 and PC9 lung cancer cells were seeded into 6-well plates $\left(2 \times 10^{5} /\right.$ well $)$ and incubated overnight. For transfection beginning on the next day, the cells were treated with $50 \mathrm{nmol} / 1$ siRNA or only a siRNA-Mate transfection reagent (GenePharma, Jiangsu, China) as a negative control (NC) for $48 \mathrm{~h}$. The target sequences for si-netrin-1 \#1 and si-netrin-1 \#2 were 5'-GCAAGAAGUUCGAAGUGACTT-3' and 5'-UCCAGCAGCGUGAGAAGAATT-3', respectively.

Western blot analysis. Total protein was extracted from the cells using radioimmunoprecipitation assay buffer (Cell Signaling Technology, Danvers, MA, USA), and a BCA protein assay kit (Abcam, Cambridge, UK) was used to determine the concentration of total protein. Proteins were then separated by $12 \%$ sodium dodecyl sulfate-polyacrylamide gel electrophoresis and transferred to a polyvinylidene fluoride membrane. After blocking with a $5 \%$ milk solution in $1 \mathrm{X}$ Tris-buffered saline containing $0.1 \%$ Tween-20 (TBST) for $1 \mathrm{~h}$ at room temperature, the membranes were incubated with primary rabbit monoclonal antibodies to netrin-1 (dilution 1:100 cat. no. BA1671-1 ProteinTech Group, Inc., Chicago, IL, USA), E-cadherin (dilution 1:500 cat. no. 28874-1-AP ProteinTech Group, Inc.), vimentin (dilution 1:4,000 cat. no. 10366-1-AP ProteinTech Group, Inc.), AKT (dilution 1:400 cat. no. ab81238 Abcam), or $\beta$-actin (dilution 1:5,000 cat. no. 20536-1-AP ProteinTech Group, Inc.) overnight at $4^{\circ} \mathrm{C}$. After three washes with TBST buffer, the membranes were incubated with the corresponding secondary antibodies (anti-rabbit $\mathrm{IgG}$, dilution 1:10,000 cat. no. SA00001-2, ProteinTech Group, Inc.) for $1 \mathrm{~h}$ at room temperature.

Antibody binding signals were visualized using the Super Signal West Pico kit (Thermo Fisher Scientific Inc., Anthem, AZ, USA), and quantitative densitometric analysis was then performed using Eagle Eye II software (Eagle Eye Technology Ltd., London, UK). Quantification of the intensities of the immunoreactive bands was performed by the Gel Doc ${ }^{\mathrm{TM}}$
XR imaging system (Bio-Rad Laboratories, Inc., Hercules, CA, USA) and QuantityOne (Bio-Rad Laboratories, Inc.). Experiments were repeated three times, and the expression level of each target protein was normalized to the corresponding expression level of $\beta$-actin.

Microfluidic chip assay. A three-dimensional (3D) microfluidic chip (Fig. 1A and B) was constructed for use in in vitro assays to investigate the invasive behavior of A549 and PC9 cells under hypoxic conditions (27). The microchip was fabricated with poly-dimethylsiloxane (Sylgard 184; Dow Chemical, Midland, MI, USA). This device contains four units composed of three parts each, including two lateral media micro-channels and one central gel micro-channel. Cancer cells were cultured in suspension within the two media channels, which are separated from the central gel channel by micro-columns with micro-gaps that allow diffusion of chemoattractants from the Matrigel. Cell invasion was evaluated after culture of the cells within the assay system for $48 \mathrm{~h}$ at $37^{\circ} \mathrm{C}$ in $5 \% \mathrm{CO}_{2}$. For each condition, three independent experiments were performed.

Transwell assay. Cell culture inserts $(8 \mu \mathrm{m}$; BD-Falcon, Franklin Lakes, NJ, USA) were used in a Transwell assay to investigate the invasive behavior of A549 and PC9 cells under hypoxic conditions. The upper chambers were coated with $100 \mu 1$ Matrigel (Becton, Dickinson and Company, Franklin, Lakes, NJ, USA; 1:1 diluted in phosphate-buffered saline). Cells exposed to the indicated hypoxic conditions were seeded into the upper chamber, and $\sim 0.5 \mathrm{ml}$ of culture medium containing $10 \%$ FBS was added to the lower chamber. After $24 \mathrm{~h}$, cells still present on the upper membrane were gently removed with a cotton swab. Cells that had migrated to the lower surface of the membrane were fixed in $4 \%$ paraformaldehyde and then stained with $0.1 \%$ crystal violet solution (Abcam) according to the manufacturer's instructions. The mean numbers of migrating cells were calculated from five random fields under the microscope.

Wound healing assay. To analyze cell migration under various conditions, A549 and PC9 cells (5x10\% well) were grown in 6-well Petri dishes under conditions of hypoxia or normoxia. After $6 \mathrm{~h}$ of serum starvation, a scratch was made in each well using a sterile $100-\mu \mathrm{l}$ pipette tip. After $24 \mathrm{~h}$, a digital camera connected to an inverted microscope (Nikon TE200; Nikon Corp., Tokyo, Japan) was used to capture images. The experiments were performed in triplicate, and representative images are shown.

Patient sample collection. Serum samples were collected from NSCLC patients and healthy donors at the Second Affiliated Hospital of Dalian Medical University (Dalian, China) between January 2016 and May 2017. The present study was approved by the Institutional Review Boards of the Second Affiliated Hospital of Dalian Medical University and all procedures were performed according to the principles outlined in the Declaration of Helsinki. All participants provided written informed consent to the use of their tissues for the purposes of the present study. Prior to any surgical or drug treatment, peripheral blood samples were collected. These samples were 
A

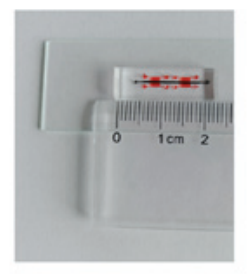

B

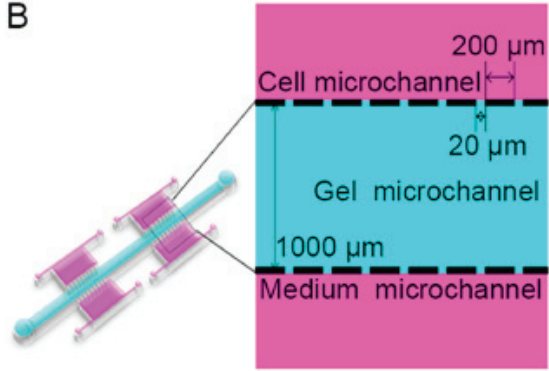

Figure 1. Layout of the prepared microfluidic chip. (A) Photograph and (B) schematic illustration. Two micro-channels are located on either side of the chip, separated by a gel micro-channel with two rows of micro-gaps. Cells were plated in the micro-channels in $20 \%$ fetal bovine serum (purple), and the gel micro-channel was filled with Matrigel (blue).

\section{A}

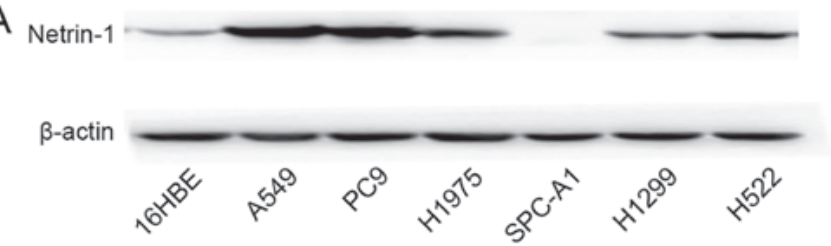

B

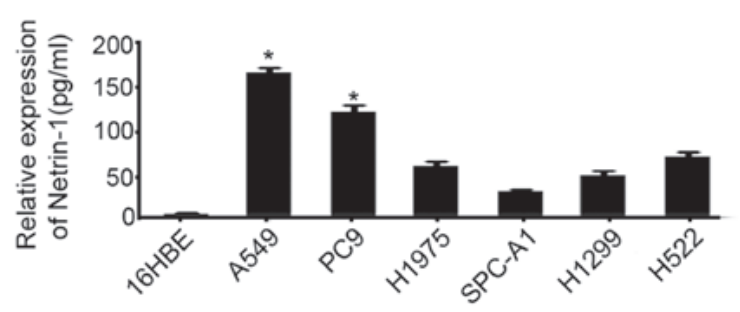

Figure 2. Netrin-1 expression in the A549, PC9, NCI-H1975, SPC-A1, NCI-H1299 and NCI-H522 lung adenocarcinoma cell lines compared with $16 \mathrm{HBE}$ cells as measured by (A) western blotting and (B) ELISA. ${ }^{*} \mathrm{P}<0.05$.

then centrifuged to obtain serum samples, which were stored at $-80^{\circ} \mathrm{C}$ until analysis. The clinical characteristics of all participants, including age, sex, smoking history and pathological results, were recorded.

ELISA. Netrin-1 concentrations in the conditioned media of cells treated under various conditions, as well as in human serum samples, were detected using a netrin-1 ELISA kit (Cusabio, Houston, TX, USA) following the manufacturer's instructions.

Statistical analysis. Data are expressed as mean \pm standard deviation. The SPSS v. 20 statistical software package (IBM Corp., Armonk, NY, USA) was used to perform Student's t-test, analysis of variance with least significant difference test or $\chi^{2}$ test to assess differences among data. A P-value $<0.05$ was considered to indicate a statistically significant difference.

\section{Results}

A549 and PC9 cells express high levels of netrin-1. Netrin-1 expression in the 16HBE, A549, NCI-H1299, NCI-H1975, SPC-A1, PC9 and NCI-H522 cell lines was examined by western blotting (Fig. 2A) and ELISA, using medium collected after culture for $24 \mathrm{~h}$ (Fig. 2B). The results demonstrated that netrin-1 was expressed in most of these lung cancer cell lines, and its expression was the highest in A549 and PC9 cells.

A549 and PC9 cells undergo EMT upon exposure to hypoxic conditions. Following exposure to $100 \mu \mathrm{M} \mathrm{CoCl}_{2}$ for $24-48 \mathrm{~h}$ to create hypoxic culture conditions, the morphology of A549 and PC9 cells was characterized by marked stretching and elongation (Fig. 3A). The epithelial biomarker E-cadherin was downregulated in these cells after exposure to hypoxia, whereas expression of the mesenchymal biomarker vimentin was increased $(\mathrm{P}<0.05$; Fig. 3B). In both the Transwell and wound healing assays, we found that the invasion (Fig. 3D and E) and migration (Fig. 3C) capacities of A549 and PC9 cells were increased in the hypoxic microenvironment. These findings represent typical events that occur during EMT of lung adenocarcinoma cells under hypoxia.

Netrin-1 expression increases in A549 and PC9 cells under hypoxic conditions. Netrin-1 expression in A549 and PC9 cells was measured following exposure of the cells to normoxia or hypoxia for $48 \mathrm{~h}$. Netrin-1 expression was significantly higher in A549 and PC9 cells cultured under hypoxic conditions compared with its levels in cells cultured under normoxic conditions $(\mathrm{P}<0.05$; Fig. $4 \mathrm{~A}$ and $\mathrm{B})$.

Netrin-1 silencing inhibits hypoxia-induced EMT of A549 and PC9 cells. To investigate the role of Netrin-1 in hypoxia-induced EMT, A549 and PC9 cells were transfected with two netrin-1 siRNAs to effectively knock down netrin-1 expression ( $\mathrm{P}<0.05$; Fig. 5A). With knockdown of netrin-1, the hypoxia-triggered EMT of A549 and PC9 cells was attenuated, with restoration of E-cadherin and vimentin expression (Fig. 5B) upon exposure of the cells to hypoxia. The Transwell (Fig. 5D) and microfluidic chip assays (Fig. 5E and F) revealed that the hypoxia-induced invasive behavior of A549 and PC9 cells was notably suppressed with knockdown of netrin-1 expression. In addition, netrin-1 silencing effectively abrogated the effects of hypoxia on the migratory behavior of these cells (Fig. 5C). Taken together, these results indicate that netrin-1 plays an important role in hypoxia-induced EMT and subsequent invasion and migration of lung cancer cells.

By comparison, under normoxic conditions, E-cadherin and vimentin expression in A549 and PC9 cells was not obviously altered by silencing of netrin- 1 expression $(\mathrm{P}>0.05$; Fig. 6A). Moreover, only slight differences in the invasive behavior of A549 and PC9 cells were observed with netrin-1 knockdown and culture under normoxic conditions $(\mathrm{P}>0.05$; Fig. 6B and C).

Netrin-1 mediates hypoxia-induced EMT by activating the PI3K/AKT pathway. To investigate whether the role of netrin-1 in hypoxia-induced EMT of lung adenocarcinoma cells involves PI3K/AKT signaling, the expression of p-AKT was measured in A549 and PC9 cells following netrin-1 knockdown. Western blotting confirmed that knockdown of netrin-1 resulted in reduced hypoxia-induced p-AKT expression in A549 and PC9 cells $(\mathrm{P}<0.05$; Fig. 7A); however, under normoxic conditions, silencing of netrin-1 did not affect the accumulation of $\mathrm{p}-\mathrm{AKT}$ (P>0.05; Fig. 7B). Taken together, these data suggest that netrin-1 is involved in hypoxia-triggered activation of $\mathrm{p}-\mathrm{AKT}$. 

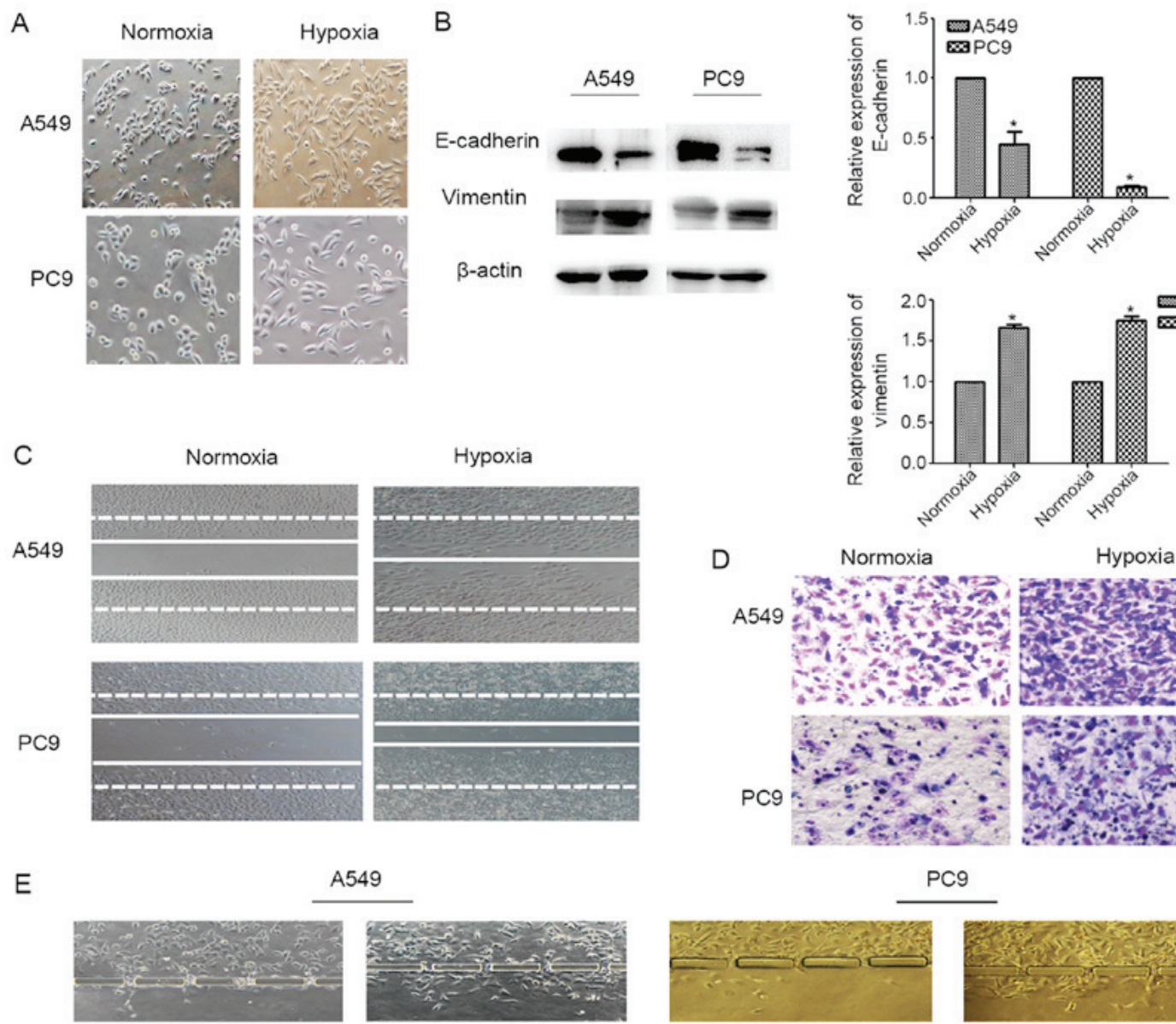

A549

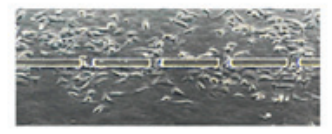

Hypoxia

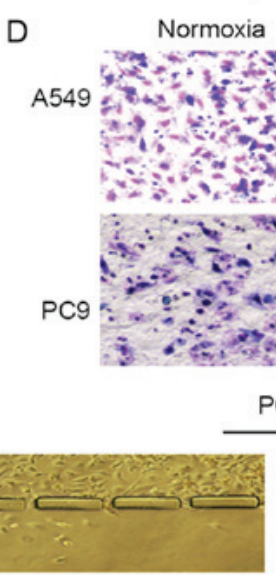

Normoxia

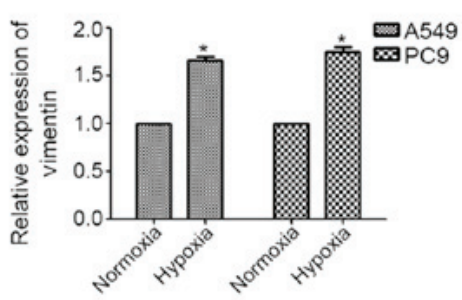

ormoxia

Figure 3. Tumor cell epithelial-to-mesenchymal transition was enhanced by $\mathrm{CoCl}_{2}$-induced hypoxia. (A) Micrographs of A549 and PC9 cells revealed the significantly stretched and elongated morphology of the cells after their culture in $100 \mu \mathrm{M} \mathrm{CoCl}_{2}$ for $24 \mathrm{~h}$. (B) Western blots showing the expression of E-cadherin and vimentin in A549 and PC9 cells following culture under hypoxic conditions. (C) Wound healing assay and (D) Transwell assay results demonstrating the cell migration and invasion abilities of A549 and PC9 cells following culture under hypoxic conditions. (E) The microfluidic assay demonstrated increased invasion of A549 and PC9 cells into the gel micro-channel following culture under hypoxic conditions. ${ }^{*} \mathrm{P}<0.05$.
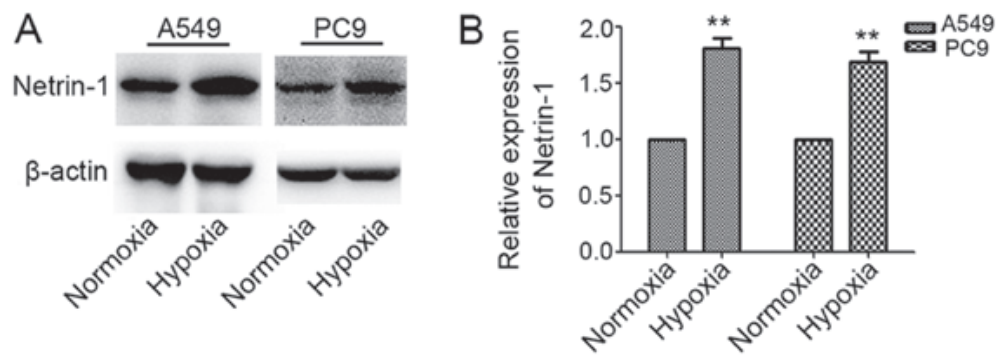

Figure 4. Netrin-1 protein expression in A549 and PC9 cells exposed to hypoxia. (A) Representative western blots and (B) quantitative analysis. "P<0.05.

Netrin-1 concentration is elevated in the serum of NSCLC patients. The concentration of netrin-1 in serum samples from 95 NSCLC patients and 95 control donors was first analyzed by ELISA. The patients' clinical data are summarized in Table I. There was no significant difference in age or sex between cancer patients and healthy control participants $(\mathrm{P}>0.05)$. Moreover, no correlations were found between the basal netrin-1 level and patient age, sex or histopathological subtype $(\mathrm{P}>0.05$; Table II), apart from TNM stage $(\mathrm{P}<0.05$; Table II). The serum concentration of netrin-1 was higher in NSCLC patients compared with that in control participants
(570.29 \pm 314.46 vs. $259.96 \pm 152.37$ pg/ml, respectively, $\mathrm{P}<0.05$; Fig. 8 and Table II).

\section{Discussion}

With $>1.61$ million new cases of lung cancer diagnosed each year and an estimated 1.59 million deaths annually worldwide (28), lung cancer has a high mortality rate that is likely due to early metastasis (29). However, the mechanisms underlying lung cancer metastasis remain to be fully elucidated. Over the past decade, extensive research on 

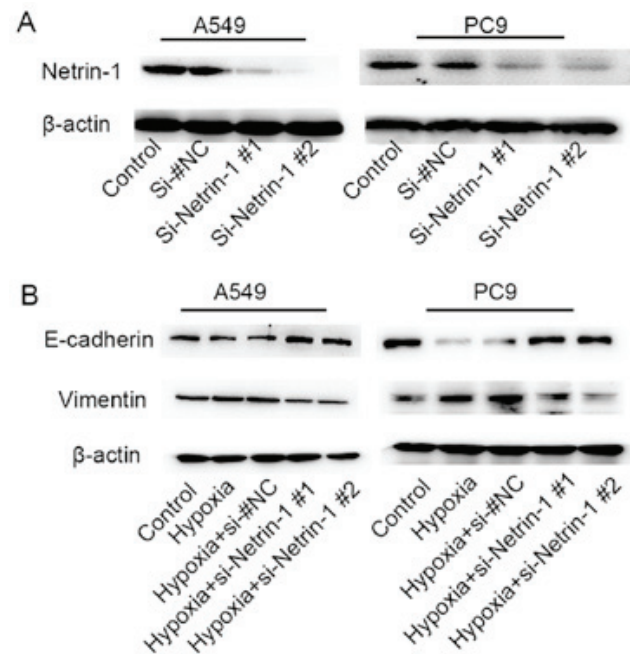

C

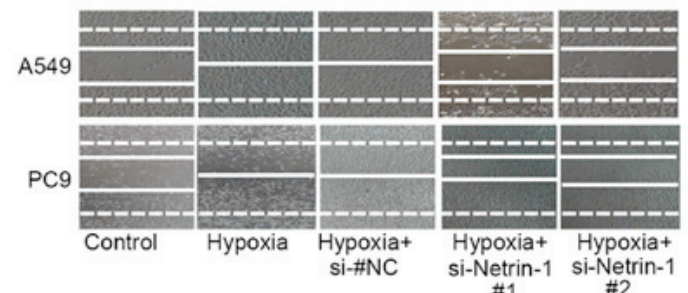

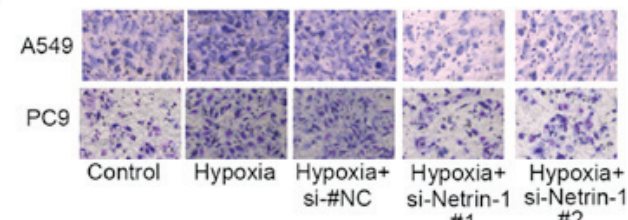

E

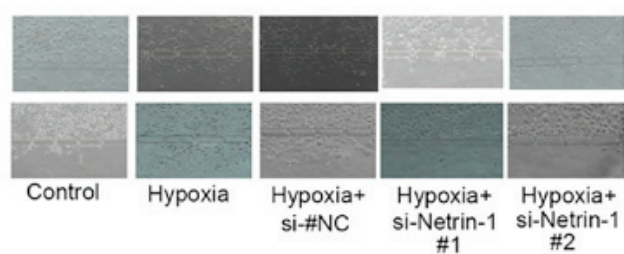

$\mathrm{F}$

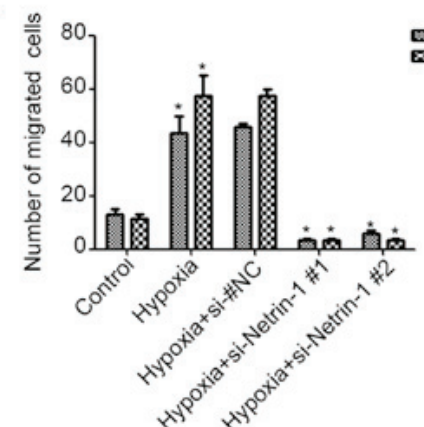

Figure 5. Netrin-1 silencing inhibited hypoxia-induced epithelial-to-mesenchymal transition of A549 and PC9 cells. (A) Netrin-1 expression in A549 and PC9 cells following transfection with siRNA (si-netrin-1 \#1 and si-netrin-1 \#2) or negative control (si-NC) and subsequent exposure to hypoxia for 24 h. Western blot analysis of (B) E-cadherin and vimentin expression in these cells. (C) Hypoxia-induced migration and invasion of A549 and PC9 cells after netrin-1 knockdown in the (D) Transwell and (E and F) microfluidic chip assay. ${ }^{*} \mathrm{P}<0.05$.

A
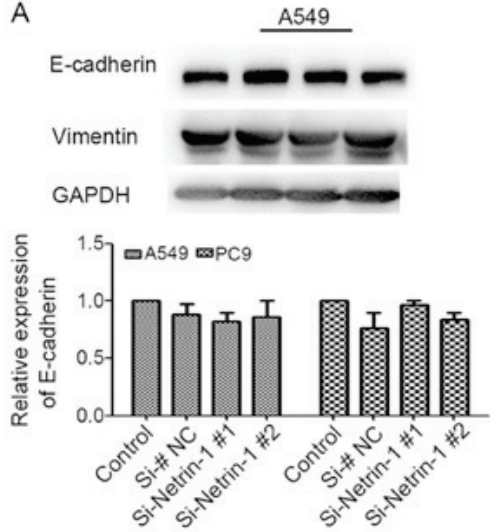

B
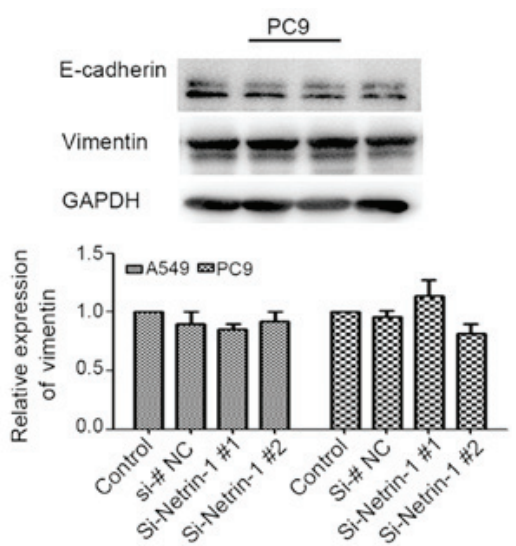

s

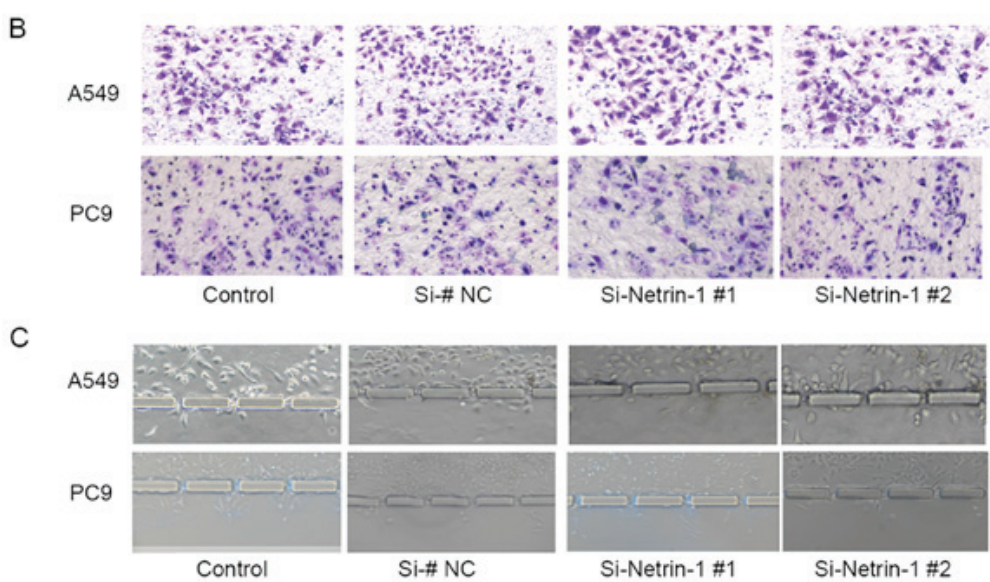

Figure 6. Netrin-1 silencing did not affect A549 and PC9 cell epithelial-to-mesenchymal transition under normoxic conditions. (A) Western blot analysis of E-cadherin and vimentin expression in these cells. Invasive behavior of these cells in the (B) Transwell and (C) microfluidic chip assay. 


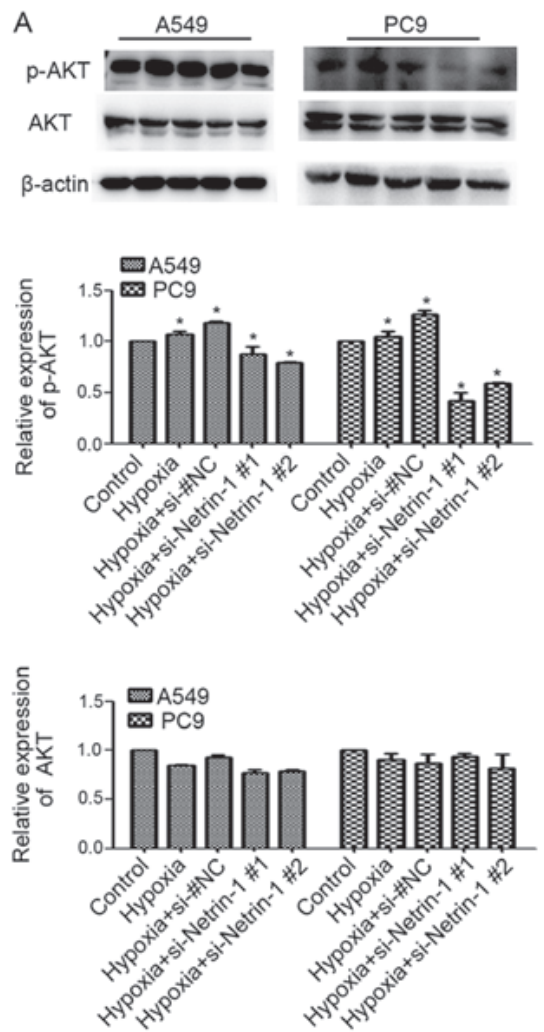

B
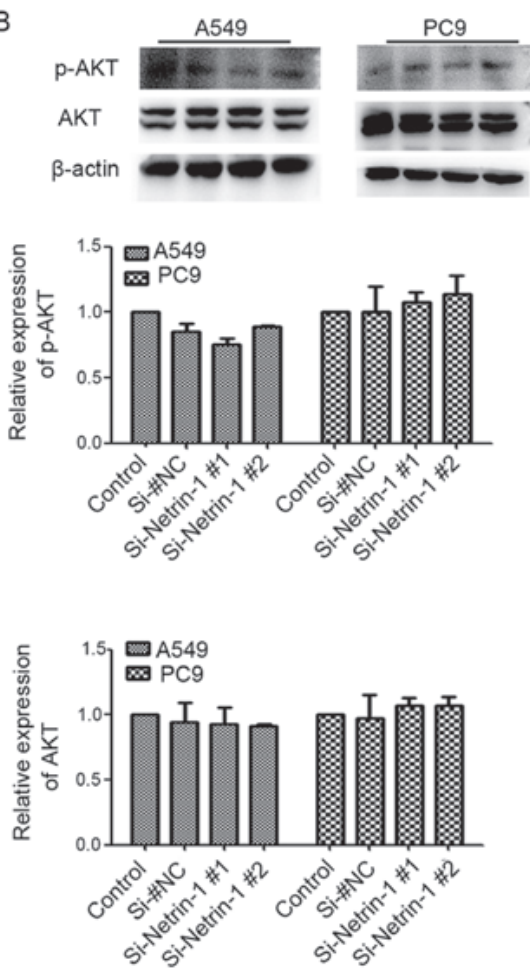

Figure 7. Netrin-1 was involved in hypoxia-induced activation of phosphoinositide 3 kinase/AKT signaling. Western blot analysis of the levels of (A) p-A KT and AKT in A549 and PC9 after netrin-1 silencing and subsequent exposure to hypoxia for $24 \mathrm{~h}$, in comparison to the levels of (B) p-AKT and AKT in these cells under normoxic conditions. ${ }^{*} \mathrm{P}<0.05$.

lung cancer has focused on cancer cells, while overlooking the unique but complex tumor microenvironment $(7,30)$. Interestingly, both experimental and clinical studies have recently reported that hypoxia within tumors may contribute to the invasive potential of tumor cells and, thus, promote metastasis (11). In the present study, lung cancer cells were treated with $\mathrm{CoCl}_{2}$ to mimic hypoxia and the hypoxic response was then investigated $(6,30)$. A simple but effective $3 \mathrm{D}$ microfluidic chip based on an in vitro assay system was used to investigate the role of netrin-1 in the hypoxia-induced EMT of A549 and PC9 cells. The use of the microfluidic chip overcomes some of the limitations associated with previous $2 \mathrm{D}$ platforms, including subjectivity in the quantification and the inability to reflect real-time changes. Furthermore, the 3D chip is used to culture the cells, thereby mimicking cell growth in vivo, and the accuracy of the device is increased by enabling multiple repetitions of the same conditions (27). The results of the present study demonstrated that $\mathrm{CoCl}_{2}$-induced hypoxia caused changes in the morphology of A549 and PC9 cells, as well as in the expression of E-cadherin and vimentin. These findings suggest that exposure to hypoxia led to EMT in these cells, and that netrin-1 plays a key role in this process and is thus potentially implicated in the progression of NSCLC.

In recent years, netrin-1 has been shown to regulate cancer progression in a number of cancer types. For example, netrin-1 was identified as a novel candidate biomarker for ovarian carcinoma, and also as a prognostic factor for brain metastases $(31,32)$. In the present study, the expression of netrin-1 was found to be increased in response to hypoxia in
A549 and PC9 cells. Moreover, netrin-1 expression was strongly upregulated in the serum of NSCLC patients compared with control donors. Interestingly, An et al reported that netrin-1 is markedly underexpressed and suppresses the growth of cancer cells in stage I/II pancreatic ductal adenocarcinoma (33). These conflicting activities of netrin-1 may be attributed to differences in the test methods and heterogeneities between these tumor types. Moreover, upregulation of netrin-1 was shown to promote cancer cell migration and invasion in human hepatocellular carcinoma (34). We observed that knockdown of netrin-1 using si-RNA significantly reduced the invasiveness and EMT of lung cancer cells in a simulated hypoxic environment. These data indicate that netrin-1 was activated in hypoxia-induced EMT. Furthermore, NP137, the first humanized netrin-1 antibody generated, was found to have antitumor activity both in solid tumors and hematological malignancies in a preclinical trial (35). Those findings indicate a direct association of netrin-1 activation with hypoxia-induced EMT, and suggest its potential as a therapeutic target for lung cancer treatment.

Several classical signaling pathways, including the Notch, Wnt and PI3K/AKT pathways, are involved in hypoxia-induced EMT (36-38), but the underlying molecular mechanisms remain unclear. Netrin-1 was shown to promote cell migration and tube formation mediated by activation of the FAK/PI3K/AKT signaling pathway in hypopharyngeal cancer (24). Zhang et al also suggested that netrin-1 may act as a pro-metastatic factor in NSCLC by enhancing cell invasion and migration via PI3K/AKT-mediated EMT (39). The aim of the present study was to determine whether netrin-1 promotes 
Table I. Clinical characteristics of 95 patients with non-small cell lung cancer and 95 healthy controls.

\begin{tabular}{lccc}
\hline Characteristics & $\begin{array}{c}\text { Patients } \\
(\mathrm{n}=95)\end{array}$ & $\begin{array}{c}\text { Controls } \\
(\mathrm{n}=95)\end{array}$ & P-value $^{\mathrm{a}}$ \\
\hline Age, years & & & 0.146 \\
$\leq 60$ & 55 & 45 & \\
$>60$ & 40 & 50 & 0.374 \\
Sex & & & \\
Male & 54 & 60 & \\
Female & 41 & 35 & \\
\hline
\end{tabular}

${ }^{\mathrm{a}} \mathrm{P}<0.05$ was considered statistically significant.

Table II. Correlations between serum levels of netrin-1 and clinicopathological characteristics of patients with non-small cell lung cancer $(\mathrm{n}=95)$.

\begin{tabular}{lccc}
\hline Characteristics & $\begin{array}{c}\text { Patients } \\
(\mathrm{n}=95)\end{array}$ & $\begin{array}{c}\text { Netrin-1 } \\
(\text { mean } \pm \text { SD })\end{array}$ & P-value $^{\mathrm{a}}$ \\
\hline Cancer patients & 55 & $570.39 \pm 314.46$ & 0.00 \\
Healthy subjects & 40 & $258.66 \pm 153.74$ & \\
Age, years & & & 0.574 \\
$\leq 60$ & 54 & $548.84 \pm 261.75$ & \\
$>60$ & 41 & $585.88 \pm 349.38$ & \\
Sex & & & 0.253 \\
Male & 72 & $545.03 \pm 291.08$ & \\
Female & 23 & $603.56 \pm 343.69$ & \\
Stage & & & 0.03 \\
I+II & 69 & $628.98 \pm 319.06$ & \\
III+IV & 26 & $422.46 \pm 251.95$ & \\
Differentiation & & & 0.143 \\
Low & 17 & $671.79 \pm 401.71$ & \\
Moderate + high & 78 & $548.16 \pm 290.55$ & \\
Histological type & & & 0.686 \\
Adenocarcinoma & 80 & $575.97 \pm 304.50$ & \\
Squamous cell & 15 & $539.95 \pm 373.58$ & \\
carcinoma & & & \\
\hline
\end{tabular}

${ }^{\mathrm{a}} \mathrm{P}<0.05$ was considered statistically significant; SD, standard deviation.

hypoxia-induced EMT through activation of the PI3K/AKT pathway. We observed that $\mathrm{p}-\mathrm{AKT}$, a critical protein in the PI3K/AKT pathway, was upregulated during hypoxia-induced EMT of A549 and PC9 cells (40). Moreover, knockdown of netrin-1 led to reduced expression of p-AKT under hypoxic conditions and indirectly inhibited tumor cell migration and invasion. Furthermore, these effects of netrin-1 silencing were not observed in A549 and PC9 cells cultured under normoxic conditions. A limitation of the present study is that the mechanism through which netrin-1 promotes EMT via

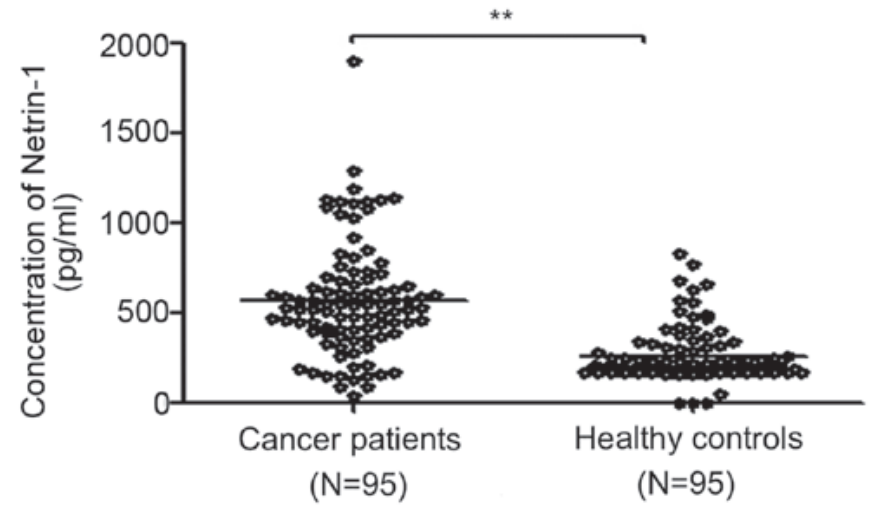

Figure 8 . The serum concentration of netrin-1 was higher in patients with non-small cell lung cancer compared with that in healthy volunteers. ${ }^{* *} \mathrm{P}<0.01$.

the PI3K/AKT pathway has yet to be verified by upregulating netrin-1 expression in lung cancer cells.

In conclusion, the results of the present study provide evidence that netrin-1 promotes hypoxia-induced EMT in lung cancer cells via the PI3K/AKT pathway in vitro. The analysis of the patients' serum samples also revealed that netrin-1 may serve as a useful clinical biomarker for predicting lung cancer progression. Therefore, netrin-1 may represent a novel therapeutic target for preventing lung cancer progression and metastasis.

\section{Acknowledgements}

The authors would like to thank the staff of the laboratory for their help in carrying out the present study.

\section{Funding}

This study was supported by a grant from the Natural Science Foundation of Liaoning Province (no. 20170540242).

\section{Availability of data and materials}

All the datasets generated/analyzed in the present study are available from the corresponding author on reasonable request.

\section{Authors' contributions}

XYJ designed the study, completed all experiments, and wrote and revised the manuscript. HQL appraised relevant studies and assisted with drafting and revising the manuscript. $\mathrm{HC}$ performed data analysis and figure preparation. LNY and JZ appraised relevant studies. QW participated in conceiving and designing the study, LHC conceived and designed the study, and assisted with drafting and revising the manuscript. All the authors have read and approved the final version of this manuscript for publication.

\section{Ethics approval and consent to participate}

The present study was approved by the Institutional Review Boards of the Second Affiliated Hospital of Dalian Medical University and all procedures were performed according to the 
principles outlined in the Declaration of Helsinki. All participants provided written informed consent regarding the use of their sera for research purposes.

\section{Patient consent for publication}

Not applicable.

\section{Competing interests}

All the authors declare that they have no competing interests to disclose.

\section{References}

1. Torre LA, Bray F, Siegel RL, Ferlay J, Lortet-Tieulent J and Jemal A: Global cancer statistics, 2012. CA Cancer J Clin 65: 87-108, 2015

2. Shaikh D, Zhou Q, Chen T, Ibe JC, Raj JU and Zhou G: cAMP-dependent protein kinase is essential for hypoxia-mediated epithelial-mesenchymal transition, migration, and invasion in lung cancer cells. Cell Signal 24: 2396-2406, 2012.

3. Gunther S, Ostheimer C, Stangl S, Specht HM, Mozes P, Jesinghaus M, Vordermark D, Combs SE, Peltz F, Jung MP, et al: Correlation of Hsp70 Serum Levels with Gross Tumor Volume and Composition of Lymphocyte Subpopulations in Patients with Squamous Cell and Adeno Non-Small Cell Lung Cancer. Front Immunol 6: 556, 2015.

4. Foss KM, Sima C, Ugolini D, Neri M, Allen KE and Weiss GJ: miR-1254 and miR-574-5p: serum-based microRNA biomarkers for early-stage non-small cell lung cancer. J Thorac Oncol 6: 482-488, 2011

5. Wang S, Li E, Gao Y, Wang Y, Guo Z, He J, Zhang J, Gao Z and Wang Q: Study on invadopodia formation for lung carcinoma invasion with a microfluidic 3D culture device. PLoS One 8: e56448, 2013.

6. Zhao M, Zhang Y, Zhang H, Wang S, Zhang M, Chen X, Wang H, Zeng G, Chen X, Liu G, et al: Hypoxia-induced cell stemness leads to drug resistance and poor prognosis in lung adenocarcinoma. Lung Cancer 87: 98-106, 2015.

7. Ye LY, Chen W, Bai XL, Xu XY,Zhang Q, Xia XF, Sun X, Li GG, $\mathrm{HuQD}, \mathrm{FuQH}$, etal:Hypoxia-Induced Epithelial-to-Mesenchymal Transition in Hepatocellular Carcinoma Induces an Immunosuppressive Tumor Microenvironment to Promote Metastasis. Cancer Res 76: 818-830, 2016.

8. Zhang L, Huang G, Li X, Zhang Y, Jiang Y, Shen J, Liu J, Wang Q, Zhu J, Feng X, et al: Hypoxia induces epithelial-mesenchymal transition via activation of SNAI1 by hypoxia-inducible factor-1 $\alpha$ in hepatocellular carcinoma. BMC Cancer 13: 108, 2013.

9. Chen S, Chen JZ, Zhang JQ, Chen HX, Yan ML, Huang L, Tian YF, Chen YL and Wang YD: Hypoxia induces TWIST-activated epithelial-mesenchymal transition and proliferation of pancreatic cancer cells in vitro and in nude mice. Cancer Lett 383: 73-84, 2016.

10. Masoud GN and Li W: HIF-1 $\alpha$ pathway: Role, regulation and intervention for cancer therapy. Acta Pharm Sin B 5: 378-389, 2015.

11. Vaupel P and Multhoff G: Accomplices of the Hypoxic Tumor Microenvironment Compromising Antitumor Immunity: Adenosine, Lactate, Acidosis, Vascular Endothelial Growth Factor, Potassium Ions, and Phosphatidylserine. Front Immunol 8: $1887,2017$.

12. Nakamura H, Ichikawa T, Nakasone S, Miyoshi T, Sugano M, Kojima M, Fujii S, Ochiai A, Kuwata T, Aokage K, et al: Abundant tumor promoting stromal cells in lung adenocarcinoma with hypoxic regions. Lung Cancer 115: 56-63, 2018.

13. Dominici C, Moreno-Bravo JA, Puiggros SR, Rappeneau Q, Rama N, Vieugue P, Bernet A, Mehlen P and Chédotal A: Floor-plate-derived netrin-1 is dispensable for commissural axon guidance. Nature 545: 350-354, 2017.

14. Xu K, Wu Z, Renier N, Antipenko A, Tzvetkova-Robev D, Xu Y, Minchenko M, Nardi-Dei V, Rajashankar KR, Himanen J, et al: Neural migration. Structures of netrin-1 bound to two receptors provide insight into its axon guidance mechanism. Science 344: $1275-1279,2014$
15. Dun XP and Parkinson DB: Role of Netrin-1 Signaling in Nerve Regeneration. Int J Mol Sci 18: 18, 2017.

16. Chen J, Du H, Zhang Y, Chen H, Zheng M, Lin P, Lan Q, Yuan Q, Lai Y, Pan X, et al: Netrin-1 Prevents Rat Primary Cortical Neurons from Apoptosis via the DCC/ERK Pathway. Front Cell Neurosci 11: 387, 2017.

17. Bai L, Mei X, Wang Y, Yuan Y, Bi Y, Li G, Wang H, Yan P and Lv G: The Role of Netrin-1 in Improving Functional Recovery through Autophagy Stimulation Following Spinal Cord Injury in Rats. Front Cell Neurosci 11: 350, 2017.

18. Ko SY, Blatch GL and Dass CR: Netrin-1 as a potential target for metastatic cancer: Focus on colorectal cancer. Cancer Metastasis Rev 33: 101-113, 2014

19. Lv J, Sun X, Ma J, Ma X, Zhang Y, Li F, Li Y and Zhao Z: Netrin-1 induces the migration of Schwann cells via p38 MAPK and PI3K-Akt signaling pathway mediated by the UNC5B receptor. Biochem Biophys Res Commun 464: 263-268, 2015.

20. Akino T, Han X, Nakayama H, McNeish B, Zurakowski D, Mammoto A, Klagsbrun M and Smith E: Netrin-1 promotes medulloblastoma cell invasiveness and angiogenesis, and demonstrates elevated expression in tumor tissue and urine of patients with pediatric medulloblastoma. Cancer Res 74: 3716-3726, 2014.

21. Grandin M, Mathot P, Devailly G, Bidet Y, Ghantous A, Favrot C, Gibert B, Gadot N, Puisieux I, Herceg Z, Delcros JG, Bernet A and Mehlen P: Inhibition of DNA methylation promotes breast tumor sensitivity to netrin-1 interference. 8: 863-877, 2016.

22. Wang H, Zhang B, Gu M, Li S, Chi Z and Hao L: Overexpression of the dependence receptor UNC5H4 inhibits cell migration and invasion, and triggers apoptosis in neuroblastoma cell. Tumour Biol 35: 5417-5425, 2014.

23. Gibert B and Mehlen P: Dependence Receptors and Cancer: Addiction to Trophic Ligands. Cancer Res 75: 5171-5175, 2015.

24. Zhang Y, Wang B, Chen X, Li W and Dong P: AGO2 involves the malignant phenotypes and FAK/PI3K/AKT signaling pathway in hypopharyngeal-derived FaDu cells. Oncotarget 8: 54735-54746, 2017.

25. Zhang PF, Li KS, Shen YH, Gao PT, Dong ZR, Cai JB, Zhang C, Huang XY, Tian MX, Hu ZQ, et al: Galectin-1 induces hepatocellular carcinoma EMT and sorafenib resistance by activating FAK/PI3K/AKT signaling. Cell Death Dis 7: e2201, 2016.

26. Yang X, Li S, Zhong J, Zhang W, Hua X, Li B and Sun H: CD151 mediates netrin-1-induced angiogenesis through the Src-FAK-Paxillin pathway. J Cell Mol Med 21: 72-80, 2017.

27. Song J, Zhang Y, Zhang C, Du X, Guo Z, Kuang Y, Wang Y, Wu P, Zou K, Zou L, et al: A microfluidic device for studying chemotaxis mechanism of bacterial cancer targeting. Sci Rep 8: 6394, 2018 .

28. Torre LA, Siegel RL and Jemal A: Lung Cancer Statistics. Adv Exp Med Biol 893: 1-19, 2016.

29. Quéré G, Descourt R, Robinet G, Autret S, Raguenes O, Fercot B, Alemany P, Uguen A, Férec C, Quintin-Roué I, et al: Mutational status of synchronous and metachronous tumor samples in patients with metastatic non-small-cell lung cancer. BMC Cancer 16: 210, 2016.

30. Chu CY, Jin YT, Zhang W, Yu J, Yang HP, Wang HY, Zhang ZJ, Liu XP and Zou Q: CA IX is upregulated in CoCl2-induced hypoxia and associated with cell invasive potential and a poor prognosis of breast cancer. Int J Oncol 48: 271-280, 2016.

31. Papanastasiou AD, Pampalakis G, Katsaros D and Sotiropoulou G: Netrin-1 overexpression is predictive of ovarian malignancies. Oncotarget 2: 363-367, 2011.

32. Harter PN, Zinke J, Scholz A, Tichy J, Zachskorn C, Kvasnicka HM, Goeppert B, Delloye-Bourgeois C, Hattingen E, Senft C, et al: Netrin-1 expression is an independent prognostic factor for poor patient survival in brain metastases. PLoS One 9: e92311, 2014.

33. An XZ, Zhao ZG, Luo YX, Zhang R, Tang XQ, Hao D, Zhao X, Lv X and Liu D: Netrin-1 suppresses the MEK/ERK pathway and ITGB4 in pancreatic cancer. Oncotarget 7: 24719-24733, 2016.

34. Han P, Fu Y, Liu J, Wang Y, He J, Gong J, Li M, Tan Q, Li D, Luo Y, et al: Netrin-1 promotes cell migration and invasion by down-regulation of BVES expression in human hepatocellular carcinoma. Am J Cancer Res 5: 1396-1409, 2015.

35. Ducarouge B, Delcros JG, Abès R, Goldschneider D, Gibert B, Blachier J, Neves D, Mehlen P, Bernet A and Depil S: Abstract 2921: Preclinical characteristics of NP137, a first-in-class monoclonal antibody directed against netrin-1 and inducing dependence receptors-mediated cell death. Cancer Res 75 (Suppl): 2921-2921, 2015. 
36. Fujiki K, Inamura H, Miyayama T and Matsuoka M: Involvement of Notch1 signaling in malignant progression of A549 cells subjected to prolonged cadmium exposure. J Biol Chem 292: 7942-7953, 2017

37. Hong CF, Chen WY and Wu CW: Upregulation of Wnt signaling under hypoxia promotes lung cancer progression. Oncol Rep 38: $1706-1714,2017$

38. Zhou Z, Wang S, Song $\mathrm{C}$ and $\mathrm{Hu} \mathrm{Z}$ : Paeoniflorin prevents hypoxia-induced epithelial-mesenchymal transition in human breast cancer cells. OncoTargets Ther 9: 2511-2518, 2016.
39. Zhang X, Cui P, Ding B, Guo Y, Han K, Li J, Chen H and Zhang W: Netrin-1 elicits metastatic potential of non-small cell lung carcinoma cell by enhancing cell invasion, migration and vasculogenic mimicry via EMT induction. Cancer Gene Ther 25: $18-26,2018$

40. Wang $\mathrm{H}$, Zhang $\mathrm{C}$, Xu L, Zang K, Ning Z, Jiang F, Chi H, Zhu $\mathrm{X}$ and Meng Z: Bufalin suppresses hepatocellular carcinoma invasion and metastasis by targeting HIF-1 $\alpha$ via the PI3K/AKT/mTOR pathway. Oncotarget 7: 20193-20208, 2016. 